\title{
The Construction and Simulation of the Surface-scale Target Model for Torpedo
}

\author{
WU He-long ${ }^{1, a}$, Zheng Chenyao ${ }^{2, b}$, Deng Wei ${ }^{3, c}$ \\ 93 unit of 91388 Army, Guangdong, Zhanjiang, China \\ a'qingwu321@yeah.net, ${ }^{b}$ zhchy@foxmail.com, ${ }^{c}$ chenlizhidengwei@163.com
}

Keywords: Surface-scale Target; Torpedo; Model; Simulation

\begin{abstract}
This paper explains and demonstrates a two-dimensional surface-scale model of the torpedo target.In this work,it makes scientific researches on characteristics of the surface-scale targets for torpedo using some sound reflection highlights distributed in two-dimensional scale with different strengths, also this paper builds the the surface-scale target model based on matlab platform and the target test, then it sets the simulation conditions ,and makes a detailed analysis of the echo signals of the surface-scale target in the time domain and frequency domain.
\end{abstract}

\section{Introduction}

Currently, the majority of domestic target simulate the point target of submarines, or only the linear scale target, while ignoring the reflection characteristics of the longitudinal dimension. For high frequencies, any echo of one target is superimposed by a number of sub-wave echoes. Each sub-wave echo can be seen as a scattering point waves emitted by the target, these are named highlights of the scatters. Under normal circumstances, echo of submarine targets is composed of several major sub-echoes highly reflected, the main is generally reflected by the hull, the smooth part of submarine bridge, the cross of the hull and bridge, the bow and the stern. Acoustic reflection properties of the bridge, especially horizontal, vertical dimension of orientation towards the target, have a considerable influence on the echo. This paper builds the mode of surface-scale target and makes simulation to the surface-scale target with sound reflection highlights.

\section{The Construction of the Surface-scale Target Mode for Torpedo}

For larger torpedo targets, because they have a certain dimension, the reflected echo signals generated from an elongated body with a certain distribution in the distance and azimuth. For the scale of the target, it is called highlights, or point sources. Surface-scale target model simulate the submarine's lateral, vertical acoustic reflection characteristics of two-dimensional scale.

After acoustic signal transmitted, it is reflected after a distance of the target in an ideal environment (without regarding to environmental disturbances such as noise), after the signal reaches the receiving end of the torpedo, as is known, it is reflected by the highlights, then the echo signals are formed by the superposition.

In the process of the torpedo test, the echo signal in acoustic environment is influenced by some factors, mainly the Doppler broadening or compression, the time delay and the amplitude attenuation.

As shown in Figure 1, $S$ is the initial position coordinates of the torpedo, $V_{S}$ is the velocity vector of the torpedo, $T_{i}$ is the initial position coordinate of the i-th highlight, $V_{T}$ is the velocity vector of the highlight. Torpedo transmits acoustic signal at the time of $t_{0}$, the acoustic signal reaches the $\mathrm{i}$-th highlight spending $t_{1}$, at this time, the $\mathrm{i}$-th highlight has moved to $T_{i R}$, thus $S, T_{i}$ and $T_{i R}$ make up of a vector triangle, the equation can be obtained as follows:

$T_{i R}=T_{i}+\vec{V}_{T} \cdot t_{1}$.

If the speed of sound is $c$, the equation can be obtained: 


$$
\left|T_{i R}-S\right|=\left|T_{i}+V_{T}^{\mathrm{V}} \cdot t_{1}-S\right|=c \cdot t_{1}
$$

The equation can be obtained:

$$
t_{1}=\left(\left(T_{i}-S\right) \cdot \vec{V}_{T}^{T}\right) /\left(c^{2}-\left|\vec{V}_{T}\right|^{2}\right)+\sqrt{\left(\left|T_{i}-S\right|^{2}\right) /\left(c^{2}-\left|\vec{V}_{T}\right|^{2}\right)-\left[\left(\left(T_{i}-S\right) \cdot \vec{V}_{T}^{T}\right) /\left(c^{2}-\left|\vec{V}_{T}\right|^{2}\right)\right]^{2}}
$$

It can be figured out the relative velocity of the i-th highlight towards coordinate $T_{i R}$ at the time of $t_{0}$, which is named $V_{S T_{i R}}$.

$$
V_{S T_{i R}}=\left(\left|\vec{V}_{S}\right|^{2}+\left|T_{i R}-S\right|^{2}-\left|T_{i R}-S-\vec{V}_{S}\right|^{2}\right) /\left(2 \cdot\left|\vec{V}_{S}\right| \cdot\left|T_{i R}-S\right|\right) \cdot\left|\vec{V}_{S}\right|
$$

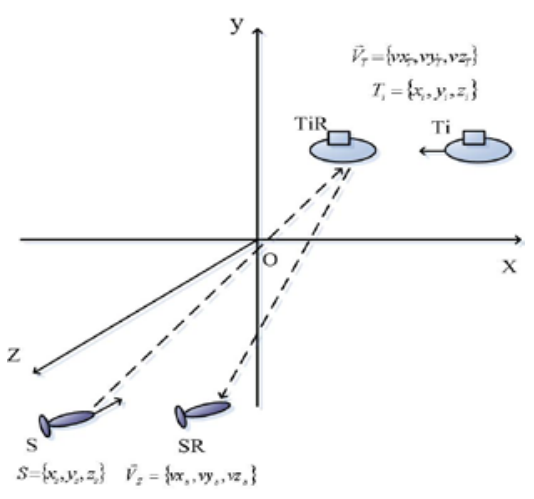

Fig. 1 The relative motion

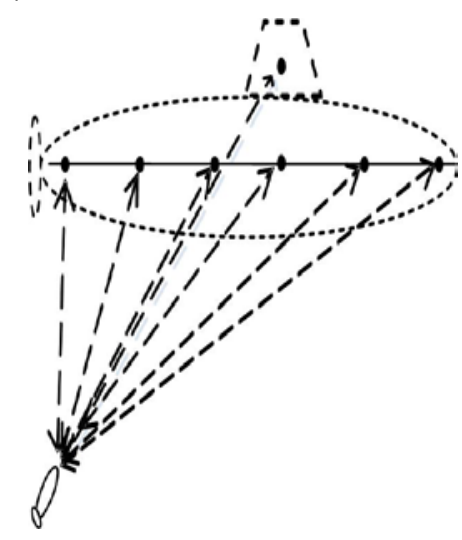

Fig. 2 The highlight mode of surface-scale target

By the same way, the relative velocity of torpedo towards coordinate $S$ at the time of $t_{1}$, which is named $V_{T_{i R} S}$.

$$
V_{T_{i R} S}=\left(\left|\vec{V}_{T}\right|^{2}+\left|T_{i R}-S\right|^{2}-\left|T_{i R}-S+\vec{V}_{T}\right|^{2}\right) /\left(2 \cdot\left|\vec{V}_{T}\right| \cdot\left|T_{i R}-S\right|\right) \cdot\left|\vec{V}_{T}\right|
$$

Torpedo pulse signal is assumed $f(t)$, then the received signal of the i-th highlight as follows:

$$
r f(t)=10^{-T L_{1} / 20} \cdot f\left(\left(t-t_{1}\right) \cdot\left(c+V_{T_{i R} S}\right) /\left(c-V_{S T_{i R}}\right)\right)
$$

$T L$ is represented to the sound propagation loss.

The echo signal is reflected by the $\mathrm{i}$-th highlight which reaches the torpedo spending $t_{2}$, then it moves to the coordinate $S_{R}$, some equations can be obtained in the vector triangle constituted of $S, S_{R}$ and $T_{i R}$ by the same way.

$$
\begin{gathered}
t_{2}=\left(\left(S+V_{S} \cdot t_{1}-T_{i R}\right) \cdot V_{S}^{T}\right) /\left(c^{2}-\left|V_{S}\right|^{2}\right)+\left(\sqrt{\left(S+V_{S} \cdot t_{1}-T_{i R}\right)^{2} \cdot\left(V_{S}^{T}\right)^{2}+\left(c^{2}-\left|V_{S}\right|^{2}\right) \cdot\left|S+V_{S} \cdot t_{1}-T_{i R}\right|^{2}}\right) /\left(c^{2}-\left|V_{S}\right|^{2}\right) \\
S_{R}=S+\vec{V}_{S} \cdot\left(t_{1}+t_{2}\right)
\end{gathered}
$$

By the law of cosines it can be calculated the relative speed what the i-th highlight moves towards coordinate $S_{R}$ at the moment $t_{1}$, which is named $V_{T_{i R} S_{R}}$.

$$
V_{T_{i R} S_{R}}=\left(\left|\vec{V}_{T}\right|^{2}+\left|T_{i R}-S_{R}\right|^{2}-\left|T_{i R}-S_{R}+\vec{V}_{T}\right|^{2}\right) /\left(2 \cdot\left|\vec{V}_{T}\right| \cdot\left|T_{i R}-S_{R}\right|\right) \cdot\left|\vec{V}_{T}\right|
$$

It can be calculated the relative speed what the torpedo moves towards coordinate $T_{i R}$ at the moment $\left(t_{1}+t_{2}\right)$, which is named $V_{S_{R} T_{i R}}$.

$$
V_{S_{R} T_{i R}}=\left(\left|\vec{V}_{S}\right|^{2}+\left|T_{i R}-S_{R}\right|^{2}-\left|T_{i R}-S_{R}-\vec{V}_{S}\right|^{2}\right) /\left(2 \cdot\left|\vec{V}_{S}\right| \cdot\left|T_{i R}-S_{R}\right|\right) \cdot\left|\vec{V}_{S}\right|
$$

The target strength of the $i$-th highlight is provided as $T S_{i}$, the echo signal torpedo received from the $i$-th highlight as follows: 


$$
\begin{aligned}
\operatorname{ef}(t) & =10^{\left(T S_{i}-T L_{2}\right) / 20} \cdot r f\left(\left(t-t_{1}-t_{2}\right) \cdot\left(c+V_{S_{R} T_{R}}\right) /\left(c-V_{T_{i R} S_{R}}\right)\right) \\
& =10^{\left(T S_{i}-T L_{1}-T L_{2}\right) / 20} \cdot f\left(\left(t-t_{1}-t_{2}\right) \cdot\left(\left(c+V_{T_{R} S}\right) /\left(c-V_{S T_{R R}}\right)\right) \cdot\left(\left(c+V_{S_{R} T_{R}}\right) /\left(c-V_{T_{R} S_{R}}\right)\right)\right)
\end{aligned}
$$

The final echoes are the superposition of all the highlights' echoes, the final echoes can be obtained as follows:

$$
R f(t)=\sum_{i=1}^{N} e f_{i}(t)
$$

It is supposed, the number of highlights for surface-scale target on the lateral hull is from 1 to $\mathrm{N}$, the number of highlights around the longitudinal shell is from 1 to $\mathrm{M}$, then the two-dimensional surface-scale target echo signal model can also be expressed as follows:

$$
R f(t)=\sum_{i=1}^{N} e f_{i}(t)+\sum_{j=1}^{M} e f_{j(t)}
$$

\section{The Echo Signal Simulation of Surface-scale Target}

The target highlights are seperated as strong and weak scattering reflective highlights, the strong highlights are distributed in the bow ,the stern, the middle part and the bridge ${ }^{[1]}$. We set six highlights for the surface-scale target mode ${ }^{[2]}$, actually, the position of the bridge is distributed in the position of $1 / 3$ to $2 / 5$ spaced the bow. Therefore, the highlight mode of surface-scale target can be constructed as the Figure 2 shown.

In Figure 2, the transverse is distributed of six to evenly highlights what each spaced 12 meters, the highlight in the longitudinal direction is set in the $2 / 5$ position from the starting point ${ }^{[3]}$, which spaced 7 meters from the transverse hull in the vertical position. Establishing a three-dimensional coordinate system $^{[4]}$, we can get the location coordinates of each highlight. Set the surface-scale target laying depth of 20 meters (assuming the same depth of torpedo), the strength of each highlight in the target $20 \mathrm{db}$, and the simulated target speed is $20 \mathrm{Knot}$ (about $10 \mathrm{~m} / \mathrm{sec}$ ), then we establish the coordinate system, the movement velocity vector of target is assumed as follows, $\vec{V}_{T}=$

$(10,0,0)$, the coordinates of each highlight around the transverse dimension as follows:

$$
\vec{T}_{1}=(0,0,-20) \quad, \vec{T}_{2}=(12,0,-20), \vec{T}_{3}=(24,0,-20), \vec{T}_{4}=(36,0,-20), \vec{T}_{5}=(48,0,-20),
$$

$\vec{T}_{6}=(60,0,-20)$, the coordinate of highlight across longitudinal dimension is: $\vec{T}_{7}=(36,7,-20)$, the initial coordinate of torpedo is: $\vec{S}=(-100,-10,-20)$, the sailing speed of torpedo is set 35Knot (about $18 \mathrm{~m} / \mathrm{sec}$ ), the velocity vector is: $\vec{V}_{S}=(18,0,0)$.

Torpedo signal is a rectangular pulse signal, the filling frequency is $20 \mathrm{KHz}$ with a unit of value, the pulse width is $100 \mathrm{~ms}$, the sampling frequency is $120 \mathrm{KHz}$, the transmission signal is expressed as $\mathrm{f}(\mathrm{t})$, the expression of the signal envelope is:

$$
x=\left\{\begin{array}{l}
0,(0<t<0.1) \\
1,(0.1<t<0.2) \\
0,(0.2<t<0.5)
\end{array}\right.
$$

The carrier is a sinusoidal signal, it is expressed as: $\mathrm{x}(\mathrm{t})=\sin (2 * \mathrm{pi} * 20000 * \mathrm{t})(<0<\mathrm{t}<0.5)$, the sampling frequency is $120 \mathrm{KHz}$, then the actual signal of torpedo is: $\mathrm{y}(\mathrm{t})=\mathrm{f}(\mathrm{t}){ }^{*} \mathrm{x}(\mathrm{t})$. Assuming that the Doppler frequency shift of target is set $0 \mathrm{KHz}$, torpedo launches at the time 0 , after time $\mathrm{t} 1$, the target receives signals by the torpedo, and then transmits signals immediately to torpedo , after the time $\mathrm{t} 2$ the torpedo receives echo signals. We can make up the expression of each of the target model parameter :

$$
\text { function }\left(T L_{1}, T L_{2}, V_{S_{R} T S_{i S}}, V_{T S_{i S} S_{R}}, V_{T R_{i R} S}, V_{S T R_{i R}}\right)=f\left(\vec{T}_{i}, \vec{V}_{T i}, \vec{S}_{i}, \vec{V}_{S i}\right)
$$

Then according to the known quantity, we can get the various echo signal parameters of highlights, and then get the echo signal. The echo signal for single highlight is as Figure 3 shown. The echo signal for the surface-scale target is as Figure 4 shown . 


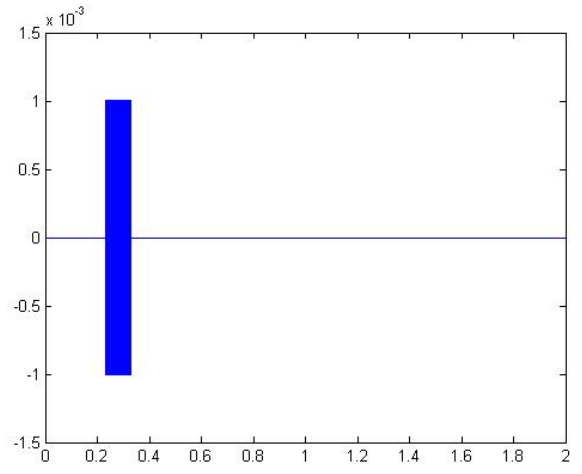

Fig.3 The echo signal waveform for single highlight

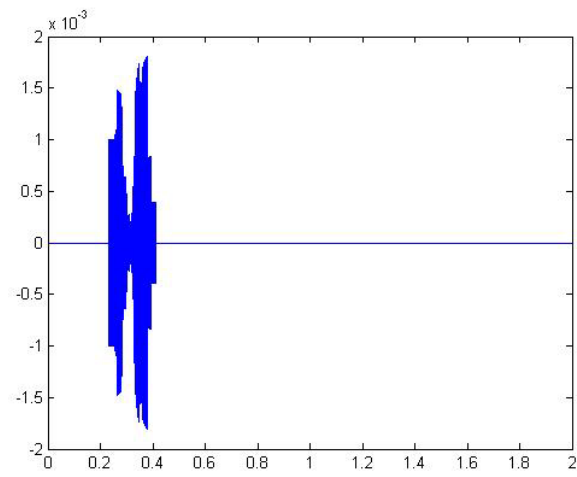

Fig.4 The echo signal waveform for the surface-scale target

Compared with Figure 3 and Figure 4, it is clearly known that the echo signal for single highlight is more regular, no changes in the amplitude of fluctuation, and the scale properties of target aren't reflected, which is due to the simulation of an ideal noiseless environment, as well as the echo for single highlight don't have the echo superposition for multiple target highlights.

The changes of target echo signal in the frequency domain are shown in Figure 5. The first figure shows a frequency-domain diagram of the original incoming signal, the second figure is a frequency domain diagram of the target echo signal. It can be seen that the relative movement between the torpedo and the target results in the Doppler shift in a certain degree, we can clearly see the changes in freuency from the comparison of the two figures.
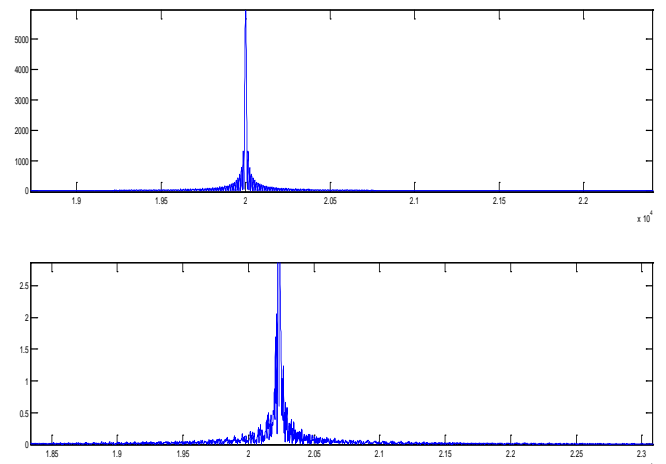

Fig.5 The comparison chart with torpedo incident signal and the echo signal

\section{Conclusion}

This paper builds the mode of surface-scale target based on the actual size of the submarine, the acoustic reflection characteristics and other parameters. It makes use of the reflection highlights with different strengths distributed in vertical and horizontal position to simulate the underwater target with a surface scale.It sets the target condition for simulation of the target in the time and frequency domain and makes a detailed analysis of the target echo signal. The simulation results show that the surface-scale target model can be more accuracy and realistic in the simulation of submarine's echoes, especially in the scale. It enhances the ability to respond to the scale identification of torpedo, so the surface-scale target can play a more important role in the sound warfare underwater in the near future.

\section{References}

[1] Li Jinming, Zhao Junwei, Wang Mingzhou, Fu Xiaohui:A High Precision Technique for Simulating Underwater Target Echo, Chinese Journal of Scientific Instrument[J], Vol.27 No.6, Jun.2006.

[2] William G, Harris J: Sonic echo simulator, United States Patent[P],Patent Number 49723379, 1990.

[3] Robert J, Grick: Principles of Underwater Sound 3d Edition[M], McGraw-Hill Book Company,1983.

[4] Zhu Lichao, Wei Pengju, Gao Jie,Tang Shuangxi, Li Guoping: Ship Electronic Engineering, Vol.31,No.11,153. 